Note

\title{
Evaluation of Antioxidative Activity for Extracts from Defatted Rice Bran Using
}

\section{5-Axe Cobweb Chart}

\author{
Yuki Sha, Tai-Ying Chiou, Takashi KobaYashi and Shuji Adachi* \\ Division of Food Science and Biotechnology, Graduate School of Agriculture, Kyoto University, Sakyo-ku, Kyoto 606-8502, Japan
}

Received April 19, 2012; Accepted July 5, 2012

Defatted rice bran was treated at $230^{\circ} \mathrm{C}$ for $5 \mathrm{~min}$ with distilled water or $40 \%$ (v/v) acetone to produce the corresponding extracts. Acetone-soluble substances were fractionated from the extracts. The antioxidative activities against 1,1-diphenyl-2-picrylhydrazyl, hydroxyl and peroxyl radicals, and the hypochlorite and peroxynitrite ions of the extracts and acetone-soluble substances were examined, and the activities were comprehensively characterized using the 5-axe cobweb chart. The $40 \%$ acetone extract exhibited higher activity than the water extract. Fractionation of acetone-soluble substances from extracts increased activity. The acetone-soluble substances from the extract with $\mathbf{4 0 \%}$ acetone exhibited the highest activity among the extracts and acetone-soluble substances.

Keywords: antioxidative activity, defatted rice bran, DPPH radical, hydroxyl radical, peroxyl radical, hypochlorite ion, peroxynitrite ion, 5-axe cobweb chart

\section{Introduction}

Rice bran is a by-product of the rice milling process used for producing white rice. Treatment with hexane produces rice bran oil, and the residue after extraction is defatted rice bran. Although it contains numerous beneficial substances, such as protein and phenolic compounds (Abdul-Hamid and Luan, 2000; Xu et al., 2001; Iqbal et al., 2005), only some of this is used for feed, while most is discarded as agrowaste.

Water that maintains its liquid state in the temperature range from 100 to $374^{\circ} \mathrm{C}$ is known as subcritical water or compressed hot water. Such water has a low relative dielectric constant similar to that of organic solvents, such as methanol and acetone, at ambient temperature (Ayala et al., 2001; Herrero et al., 2006). The water also has a high ion product (Kuhlmann et al., 1994), and acts as a catalyst for hydrolysis or degradation (Kabyemela et al., 1997; Lesutis et al., 1999; Haghighat Khajavi et al., 2006). In this context, we treated the defatted rice bran with subcritical water to produce extracts having antioxidative and emulsifying properties, depending on treatment temperature (Wiboonsirikul et al., 2007a; 2007b; 2008; Hata et al., 2008; Murayama et

*To whom correspondence should be addressed.

E-mail: adachi@kais.kyoto-u.ac.jp al., 2009; Jeyashoke et al., 2010; Chiou et al., 2011). We also reported that aqueous ethanol (Chiou et al., 2012a) and aqueous acetone (Chiou et al., 2011b) were more effective extractants than pure water under subcritical conditions for producing an extract with a higher antioxidative activity.

Evaluation of antioxidative activity using the 1,1-diphenyl-2-picrylhydrazyl (DPPH) radical is a simple and quantitative method (Blois, 1958), and has been widely used. We also used this method for evaluating the antioxidative activity of defatted rice bran extracts based on its subcritical fluid treatment in the above-cited studies. However, the physiological significance of the activity is unknown and application of methods for evaluation of the antioxidants for food use is questionable because the DPPH radical does not exist within living organisms. Terashima et al. (2010) proposed a novel method for evaluating the antioxidative activity of water-soluble antioxidants against the hydroxyl radical, the peroxyl radical, the hypochlorite ion, and the peroxynitrite ion based on the structural changes of myoglobin, as well as against DPPH radicals. The physiological functions of the reactive oxygen species have been elucidated (Valko et al., 2007). The activity of the antioxidant was comprehensively characterized using the 5-axe cobweb chart.

In this study, the defatted rice bran extracts were obtained 
by treating the bran with distilled water or $40 \%(\mathrm{v} / \mathrm{v})$ acetone under subcritical conditions based on the best results in our previous study (Chiou et al., 2012b). Acetone-soluble substances were also prepared from extracts at room temperature. The antioxidative activity of the extracts and substances were then evaluated using the method of Terashima et al. (2010).

\section{Materials and Methods}

Materials Defatted rice bran was supplied by Tsuno Food Industrial (Wakayama, Japan). Its moisture content was $12.8 \%$ on a wet basis. Acetone, L-ascorbic acid, 1,1-diphenyl-2-picrylhydrazyl (DPPH), 2,2'-azobis-2-methyl-propanimidamide dihydrochloride (AAPH; purity 95\%), L-carnosine $(98.0 \%)$, sodium hypochlorite (effective concentration $>$ $5.0 \%)$ and hydrogen peroxide $(30.0-35.5 \%)$ were purchased from Wako Pure Chemical Industries (Osaka, Japan). Gallic acid (97.5\%) and myoglobin from equine skeletal muscle (95 - 100\%) were purchased from Sigma-Aldrich Japan (Tokyo, Japan). Iron (II) sulfate heptahydrate $(99.0 \%)$ and peroxynitrite solution ( $>45 \mathrm{mmol} / \mathrm{L}$ ) were purchased from Nacalai Tesque (Kyoto, Japan) and Dojindo Laboratories (Kumamoto, Japan), respectively. All the other chemicals of special grade were obtained from Wako or Nacalai.

Subcritical fluid treatment of defatted rice bran Defatted rice bran ( $3 \mathrm{~g})$ and distilled water or $40 \%(\mathrm{v} / \mathrm{v})$ aqueous acetone $(72 \mathrm{~mL})$ were placed in a TVS-N2 pressure-resistant vessel made of SUS-316 stainless steel (30 mm I.D. $\times 165.5$ $\mathrm{mm}$ height; Taiatsu Techno, Osaka, Japan). After sealing, the vessel was heated to $230^{\circ} \mathrm{C}$ using a P5-2 mantle heater (Heater Engineer, Tokyo, Japan) and a TXN-700-B programmable temperature controller (As One, Osaka, Japan). It took about $35 \mathrm{~min}$ to reach the desired temperature. The vapor pressure of $40 \%$ acetone at $230^{\circ} \mathrm{C}$ was estimated to be 3.02 MPa by Dalton's law of partial pressures and Antoine's equation (Ambrose et al., 1974). After $5 \mathrm{~min}$, the vessel was immersed in an ice-water bath for cooling. The mixture inside the vessel was clarified by filtration through a No. 2 filter paper (Toyo Roshi, Tokyo, Japan). The extract (50 mL) was concentrated using an N-1100AN rotary evaporator (Tokyo Rika Kikai, Tokyo, Japan), then freeze-dried using an FDU1200 freeze-drier (Tokyo Rika Kikai). The extracts prepared with water and $40 \%$ acetone were then designated as the water extract and acetone extract (Fig. 1), respectively.

Recovery of acetone-soluble substances Freeze-dried extracts ( $c a .0 .8 \mathrm{~g}$ water extract; $c a .1 .1 \mathrm{~g}$ acetone extract) were placed in $20 \mathrm{~mL}$ of acetone, and the mixture was sonicated for 15 min using an AU-70C ultrasonic bath sonicator (Sogo Laboratory Glass Works, Kyoto, Japan). After the mixture was transferred to a $50-\mathrm{mL}$ polypropylene centrifu- gal tube, it was centrifuged at $8900 \mathrm{rpm}$ and $-9.0^{\circ} \mathrm{C}$ for 20 min. The supernatant was recovered in a recovery flask. The precipitate was treated again with an additional $20 \mathrm{~mL}$ of acetone as mentioned above, and the supernatant was added to the recovery flask. The acetone in the flask was removed using the rotary evaporator, and the residue was freeze-dried to obtain the acetone-soluble substances. The acetone-soluble substances from the water and acetone extracts were abbreviated as acetone-soluble-WE and acetone-soluble-AE (Fig. 1), respectively.

Measurement of antioxidative activities The DPPH radical scavenging activities of the extracts and acetone-soluble substances were measured as described by Fujinami et al. (2001). The antioxidative activities of the extracts or acetone-soluble substances against hypochlorite and peroxynitrite ions, and the hydroxyl and peroxyl radicals were also assessed according to the methods proposed by Terashima et al. (2010), which were based on the structural changes of myoglobin. Examination of the activity against hypochlorite and peroxynitrite ions, and hydroxyl and peroxyl radicals are expressed as CLO, ONOO, OH and AAPH tests, respectively.

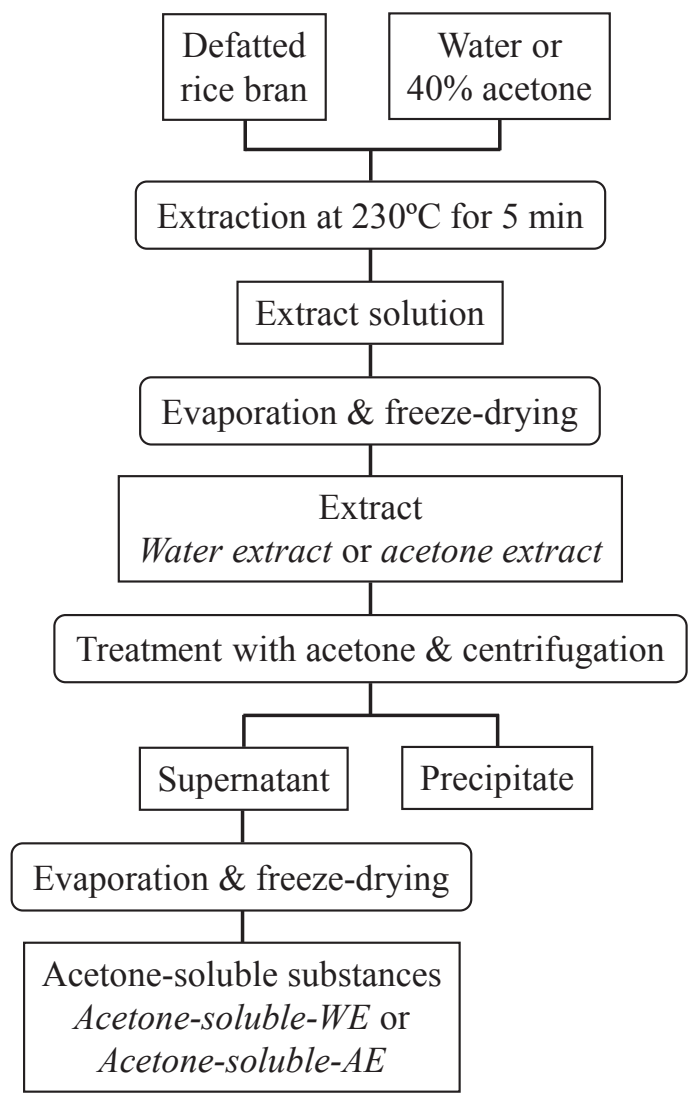

Fig. 1. Preparation of samples for evaluation of antioxidative activities. Terms expressed in italics indicate sample abbreviations. 
Statistical analysis Multiple comparison of the antioxidant activities of extracts or acetone-soluble substances was performed using Tukey's multiple range test at a significance level of $p<0.05$ using Microsoft Excel ${ }^{\circledR} 2003$.

\section{Results and Discussion}

Table 1 shows the yield of extracts from the defatted rice bran, the recovery of acetone-soluble substances, and the DPPH radical scavenging activity of the extracts and substances for treatment with water and $40 \%$ acetone. Aqueous acetone produced a higher yield of the extract than water. Both the water and acetone extracts contained acetone-soluble substances of about $35 \%$ on a weight basis. The DPPH radical scavenging activity of the acetone extract was higher than the activity of the water extract. The acetone-solubleAE had about twice the DPPH radical scavenging activity as the acetone-soluble-WE. This indicates that water in the aqueous acetone releases substances having antioxidative activity from the defatted rice bran and that these substances were solubilized by aqueous acetone.

Figure 2 shows the 5 -axe cobweb charts of the antioxidant activities for the extracts or the acetone-soluble substances against five different reactive oxygen species. For comparison, the charts for L-ascorbic acid and gallic acid with respective concentrations of $10 \mathrm{mmol} / \mathrm{L}$ and $2.5 \mathrm{mmol} /$ $\mathrm{L}$ are also shown. Although extracts prepared at room temperature are also desirable as controls, they were not tested in this study due to low yields of antioxidative substances. The water extract showed the lowest activities for the four tests, except for the AAPH test, among the extracts and substances. The acetone-soluble substances exhibited higher activities than the original extracts for both the water and acetone extracts, with the increase in activity for the $\mathrm{OH}$ test being particularly marked. The acetone-soluble-AE exhibited very high activities, exhibiting almost the same activities as the other three samples, even at half the concentration.

Because all the samples fully scavenged the AAPH radical at 10 or $5 \mathrm{~g} / \mathrm{L}$, their activities could not be compared. The

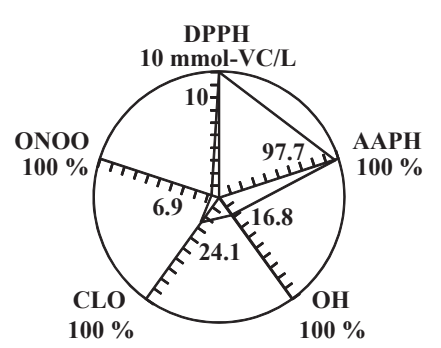

(a) L-Ascorbic acid

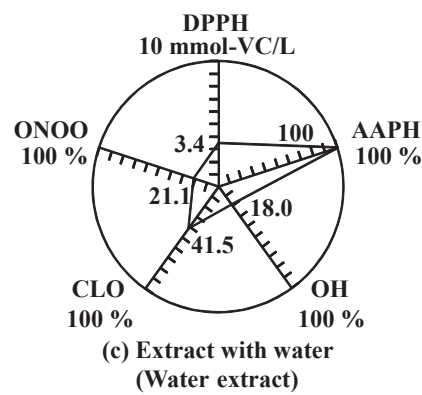

(Water extract)

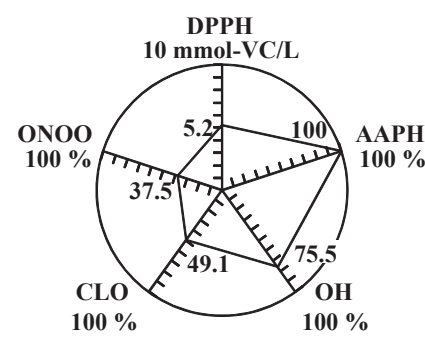

(e) Acetone-soluble substances

from water extract

(Acetone-soluble-WE)

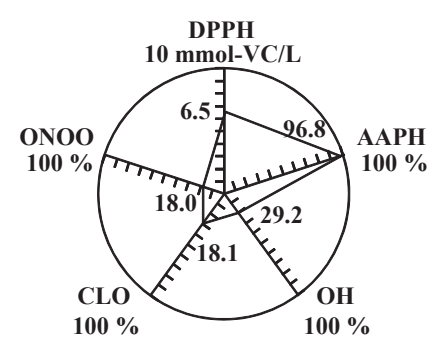

(b) Gallic acid
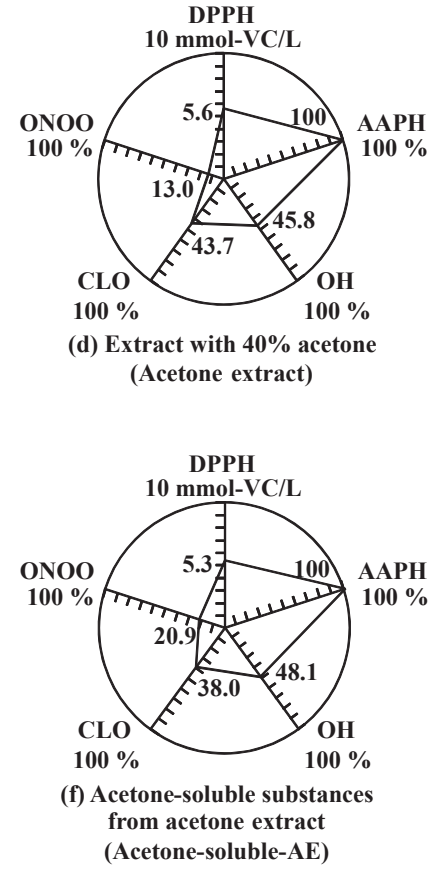

Fig. 2. 5-Axe cobweb charts for (a) L-ascorbic acid, (b) gallic acid, (c) extract from defatted rice bran with water (water extract), (d) extract from bran with $40 \%$ acetone (acetone extract), (e) acetonesoluble substances fractionated from water extract (acetone-soluble$\mathrm{WE}$ ), and (f) acetone-soluble substances fractionated from acetone extract (acetone-soluble-AE). The concentration of each sample was $10 \mathrm{~g} / \mathrm{L}$ except for the acetone-soluble-AE (5 g/L). The labels DPPH, AAPH, OH, CLO and ONOO indicate 1,1-diphenyl-2picrylhydrazyl, peroxyl radical, hydroxyl radical, hypochlorite ion and peroxynitrite ion, respectively. The abbreviation VC indicates L-ascorbic acid.

Table 1. Yield of extract from defatted rice bran by treatment with water or $40 \%(\mathrm{v} / \mathrm{v})$ acetone at $230^{\circ} \mathrm{C}$ for $5 \mathrm{~min}$, recovery of acetone-soluble substances from the extract, and the DPPH radical scavenging activities of the original extracts and acetone-soluble substances.

\begin{tabular}{lcc}
\hline & Extract with water & Extract with $40 \%$ acetone \\
\hline Yield of extract [g/g-dry bran] & $0.392 \pm 0.006$ & $0.479 \pm 0.026$ \\
Recovery of acetone-soluble substances [g/g-extract] & $0.326 \pm 0.039$ & $0.364 \pm 0.025$ \\
DPPH radical scavenging activity [mmol-VC*/g-d.m.] & & $0.560 \pm 0.014$ \\
$\quad$ Original extract & $0.345 \pm 0.015$ & $1.065 \pm 0.106$ \\
Acetone-soluble substances & $0.513 \pm 0.029$ & \\
\hline
\end{tabular}

Values are mean \pm SD for $n=3$.

* VC indicates L-ascorbic acid (vitamin C) 
CLO test indicated that the acetone-soluble-AE showed a significantly higher activity than both the water- and acetoneextracts $(p<0.05)$. Fractionation of the acetone-soluble-WE from the water-extract with acetone enhanced the activity, although the enhancement was not significant. The $\mathrm{OH}$ test indicated a significant difference for the combinations of any two samples among the four samples $(p<0.05)$. Activity against the hydroxyl radical of the acetone-soluble-WE was three times higher than that of the original water extract. The acetone extract showed lower activity on the ONOO test than the water extract. This result is different from those in other tests. However, the activity of the acetone-soluble-AE was almost the same as that of substances from the acetonesoluble-WE.

The antioxidative activity of each sample was comprehensively evaluated based on the shape of the 5-axe cobweb chart. The area of the chart relative to that of a pentagon, which was formed by connecting the five vertices of the axes, was calculated for each sample. The relative areas of the water extract, the acetone-soluble-WE, the acetone extract, and the acetone-soluble-AE were $0.15,0.40,0.27$ and 0.28 , respectively. The relative area of L-ascorbic acid and gallic acid were 0.25 and 0.22 , respectively. It should be noted that the activities of the acetone-soluble-AE were examined at half the concentration when compared to the other samples, and the concentration of gallic acid was a quarter of the concentration of L-ascorbic acid. The shape of L-ascorbic acid was similar to that of gallic acid, which is a typical phenolic compound with slightly higher hydrophobicity than Lascorbic acid. Thus, both L-ascorbic acid and gallic acid can be regarded as appropriate controls in this study. The comprehensive activity of the water extract was lower than that of L-ascorbic acid, but fractionation of the acetone-soluble substances from the extract significantly improved activity. The acetone-soluble-AE exhibited the same activity as the original acetone extract at half the concentration, and both samples showed similar profiles as gallic acid when compared with acetone-soluble-AE and the original acetone extract. This suggests that fractionation of the acetone-soluble substances concentrated the antioxidative substances present in the original extracts, and that these substances exhibited similar activities as gallic acid.

\section{References}

Abdul-Hamid, A. and Luan, Y.S. (2000). Functional properties of dietary fibre prepared from defatted rice bran. Food Chem., 68 , 15-19.

Ambrose, D., Sprake, C.H.S. and Townsend, R. (1974). Thermodynamic properties of organic oxygen compounds XXXIII. The vapour pressure of acetone. J. Chem. Thermodyn., 6, 693-700.
Ayala, R.S. and Castro, M.D.L. (2001). Continuous subcritical water extraction as a useful tool for isolation of edible essential oils. Food Chem., 75, 109-113.

Blois, M.S. (1958). Antioxidant determinations by the use of a stable free radical. Nature, 181, 1199-1200.

Chiou, T.-Y., Neoh, T.L., Kobayashi, T. and Adachi, S. (2011a). Antioxidative ability of defatted rice bran extract obtained by subcritical water extract in bulk oil and aqueous dispersion systems. Jpn. J. Food Eng., 12, 147-154.

Chiou, T.-Y., Neoh, T.L., Kobayashi, T. and Adachi, S. (2012a). Properties of extract obtained from defatted rice bran by extraction with aqueous ethanol under subcritical conditions. Food Sci. Technol. Res., 18, 37-45.

Chiou, T-Y, Neoh, T. L., Kobayashi, T. and Adachi, S. (2012b). Extraction of defatted rice bran with subcritical aqueous acetone. Biosci. Biotechnol. Biochem., in press.

Haghighat Khajavi, S., Ota, S., Kimura, Y. and Adachi, S. (2006). Kinetics of maltooligosaccharide hydrolysis in subcritical water. $J$. Agric. Food Chem., 54, 3663-3667.

Fujinami, Y., Tai, A. and Yamamoto, I (2001). Radical scavenging activity against 1,1-diphenyl-2-picryl hydrazyl of ascorbic acid 2-glucoside (AA-2G) and 6-acyl-AA-2G. Chem. Pharm. Bull., 49, 642-644.

Iqbal, S., Bhanger, M.I. and Anwar, F. (2005). Antioxidant properties and components of some commercially available varieties of rice bran in Pakistan. Food Chem., 93, 265-272.

Herrero, M., Cifuentes, A. and Ibañez, E. (2006). Sub- and supercritical fluid extraction of functional ingredients from different natural sources: plants, food-by-product, algae and microalgae. Food Chem., 98, 136-148.

Hata, S., Wiboonsirikul, J., Maeda, A., Kimura, Y. and Adachi, S. (2008). Extraction of defatted rice bran by subcritical water treatment. Biochem. Eng. J., 40, 44-53.

Jeyashoke, N., Chiou, T.-Y., Neoh, T.L., Murayama, Y., Kobayashi, T., and Adachi, S. (2010). Effect of temperature-rising rate on the antioxidative ability of the defatted rice bran extract obtained by subcritical water treatment. Food Sci. Technol. Res., 16, 197-200.

Kabyemela, B.M., Adschiri, T., Malaluan, R.M. and Arai, K. (1997). Kinetics of glucose epimerization and decomposition in subcritical and supercritical water. Ind. Eng. Chem. Res., 36, 1552-1558.

Kuhlmann, B., Amett, E.M. and Siskin, M. (1994). Classical organic reactions in pure superheated water. J. Org. Chem., 59, 30983101.

Lesutis, H.P., Gläser, R., Liotta, C.L. and Eckert, C.A. (1999). Acid/ base-catalyzed ester hydrolysis in near-critical water. Chem. Commun., 20, 2063-2064.

Murayama, Y., Neoh, T.L., Kobayashi, T. and Adachi, S. (2009). Production of emulsifying and antioxidative substances from defatted rice bran by two-step subcritical water treatment. Jpn. J. Food Eng., 10, 107-114. 
Terashima, M., Watanabe, R., Ueki, M. and Matsumura, S. (2010). Comprehensive evaluation of antioxidant activity for various substances with 5-axe cobweb chart. Food Chem., 120, 150-155.

Valko, M., Leibfritz, D., Moncol, J., Cronin, M.T., Mazur, M. and Telser, J. (2007). Free radicals and antioxidants in normal physiological functions and human disease. Int. J. Biochem. Cell Biol., 39, 44-84.

Wiboonsirikul, J., Kimura, Y., Kadota, M., Morita, H., Tsuno, T. and Adachi, S. (2007a). Properties of extracts from defatted rice bran by its subcritical water treatment. J. Agric. Food Chem., 55, 8759-8765.

Wiboonsirikul, J., Khuwijitjaru, P., Kimura, Y., Morita, H., Tsuno, T. and Adachi, S. (2007b). Production optimization of the extract with high phenolic content and radical scavenging activity from defatted rice bran by subcritical water treatment. Jpn. J. Food. Eng., 8, 311-315.

Wiboonsirikul, J., Nakazawa, R., Kobayashi, T., Morita, H., Tsuno, T. and Adachi, S. (2008). Suppression of the oxidation of methyl linoleate encapsulated with the extract from defatted rice bran by a compressed hot water treatment. Eur. Food Res. Technol., 228, 109-114.

Xu, Z., Hua, N. and Godber, J.S. (2001). Antioxidant activity of tocopherols, tocotrienols, and $\gamma$-oryzanol components from rice bran against cholesterol oxidation accelerated by 2,2'-azobis (2-methylpropionamidine) dihydrochloride. J. Agric. Food Chem., 49, 2077-2081. 